\title{
Twist modulated phases in chiral smectic liquid crystals
}

Jonathan J. Stott

Fairfield University, jstott@fairfield.edu

Rolfe G. Petschek

Follow this and additional works at: https://digitalcommons.fairfield.edu/physics-facultypubs

(C1999 American Physical Society

The final publisher PDF has been archived here with permission from the copyright holder.

\section{Peer Reviewed}

\section{Repository Citation}

Stott, Jonathan J. and Petschek, Rolfe G., "Twist modulated phases in chiral smectic liquid crystals" (1999). Physics Faculty Publications. 115.

https://digitalcommons.fairfield.edu/physics-facultypubs/115

\section{Published Citation}

Stott, Jonathan J., and Rolfe G. Petschek. "Twist modulated phases in chiral smectic liquid crystals." Physical Review E 60, no. 2 (1999): 1799.

This item has been accepted for inclusion in DigitalCommons@Fairfield by an authorized administrator of DigitalCommons@Fairfield. It is brought to you by DigitalCommons@Fairfield with permission from the rightsholder(s) and is protected by copyright and/or related rights. You are free to use this item in any way that is permitted by the copyright and related rights legislation that applies to your use. For other uses, you need to obtain permission from the rights-holder(s) directly, unless additional rights are indicated by a Creative Commons license in the record and/or on the work itself. For more information, please contact digitalcommons@fairfield.edu. 


\title{
Twist modulated phases in chiral smectic liquid crystals
}

\author{
Jonathan J. Stott and Rolfe G. Petschek \\ Department of Physics, Case Western Reserve University, Cleveland, Ohio 44106-7079 \\ (Received 27 May 1998; revised manuscript received 28 January 1999)
}

\begin{abstract}
By considering short period helical planar modulations about the layer normal, we construct a model free energy for the ferriclinic phases observed in chiral smectic liquid crystals. We then use this free energy to construct the phase diagram for our model. The resulting phases are compared with the experimentally observed smectic- $C^{*}$ subphases (ferroclinic, antiferroclinic, and heliclinic). A strong coupling is found between the ferroclinic $q=2 \pi / a$ and the heliclinic $q=2 \pi / 3 a$ modes. This coupling was not considered in previous models. The resulting additional stability of this "locked in" phase is discussed. [S1063-651X(99)10607-X]
\end{abstract}

PACS number(s): 64.70.Md, 61.30.Cz

\section{INTRODUCTION}

In a smectic liquid crystal the molecules assemble themselves into periodic layered structures. By convention, the layer normal defines the $z$ axis of the system. The molecules of the liquid crystal are anisotropic, which often leads to pronounced birefringence effects. For the systems we will consider, the molecules can be viewed as elongated ellipsoids, in which case the long 'axis of the molecule coincides with the extraordinary index of refraction.

In the smectic- $A(\mathrm{Sm}-A)$ phase, the average orientation of the long axis of the molecules is parallel to the layer normal. In the $\mathrm{Sm}-C$ phases, the orientation tilts and develops a component perpendicular to the layer normal. In the Sm- $C$ phase itself, the molecules all tilt in the same direction throughout the sample. In a chiral material, on the other hand, as one moves along the $z$ axis, the tilt direction precesses about the layer normal with a period much larger than the layer spacing. This is known as the $\mathrm{Sm}-C^{*}$ phase. Because these molecules lack a center of inversion, the $\mathrm{Sm}-C^{*}$ phase can possess a spontaneous ferroelectric polarization [1]. This polarization couples strongly to an applied electric field which has applications in the manufacture of optical devices.

Ferroclinic phases are liquid crystal phases where the average tilt vector points in the same direction from layer to layer, ignoring any rotations due to the chirality (the tilt vector is defined as the vector difference between the director and the layer normal). In antiferroclinic phases, on the other hand, the tilt vector changes direction between adjacent layers $\vec{c}_{\ell}=-\vec{c}_{\ell+1}$. Ferriclinic phases are the intermediate case where the tilt vector is neither parallel nor antiparallel to the adjacent layers. In the chiral materials we consider below, these phases also possess spontaneous polarizations; chiral ferroclinics are necessarily also ferroelectric, etc.

Subphases of the ferroclinic (ferroelectric) Sm- $C^{*}$ phase have been observed in materials such as 4-(1methylheptyloxycarbonyl) phenyl 4 - -gctylbiphenyl 4-carboxylate (MHPOBC). These subphases are known to include at least one antiferroclinic phase $\left(\mathrm{Sm}-C_{A}\right)$ as well as a ferriclinic $\left(\mathrm{Sm}-C_{\gamma}\right)$ phase and the uncharacterized $\mathrm{Sm}-C_{\alpha}$ phase. In other materials, additional ferriclinic and antiferroclinic subphases have been reported in the literature as well $[2,3]$. The $\mathrm{Sm}-\mathcal{C}_{\gamma}$ ferriclinic phase is believed $[4,5]$ to be formed from a repeating three-layer unit with two parallel and one antiparallel tilts (a " ++-++- "-type structure). Recent experimental evidence [6] suggests that other ferriclinic phases with three-layer and four-layer repeat units exist as well. To date, neither the structure of the $\mathrm{Sm}-C_{\alpha}$ phase nor the structure of the majority of the other ferriclinic phases have been experimentally determined.

Nevertheless, several models have been put forward to explain the variety of structures seen in the ferriclinic phases of chiral smectic liquid crystals. One set of models predict that the form of the ferroclinic phase(s) is a "Devil's staircase" with a formally infinite series of abrupt step-by-step changes [7-9] between the completely parallel ordering of the Sm- $C^{*}$ and the completely antiparallel ordering of the $\mathrm{Sm}-C_{A}$ phases. These models generally consider the system to be in a superposition of ferroclinic and antiferroclinic ordering and progress step by step (much like an Ising model) by rotating the polarization of an entire layer by $180^{\circ}$. The other set of models are discrete phenomenological models $[10,11]$ with competition between nearest and next-nearest neighbor interactions leading to a continuously unwinding pitch, from ferroclinic, through ferriclinic, to antiferroclinic. A variation on this general approach was proposed by Lorman $e t_{1} a l_{\text {. }}[12]$ that shows a variety of discreet phases instead of the single continuous phase.

As mentioned above, a common feature of models based on the Devil's staircase is that they assume that for lengths on the order of a few times the layer spacing the molecules always remain in a single plane: the molecules are either parallel or antiparallel. While this is certainly what is observed in the $\mathrm{Sm}-C^{*}$ and $\mathrm{Sm}-C_{A}$ phases, there is no reason to assume, a priori, that this is the only possibility. The discrete models on the other hand, while they do allow for rotations about the layer normal, have used simple expressions for the fourth order terms in the free energy and seldom show a very wide variety of possible phase transitions. To the best of our 'knowledge, no one has previously' looked at the implications of including fourth order terms that are symmetry allowed and provide ströng couplings between different Fourier components.

In this paper, we introduce a free energy that is more general than the one previously considered $[11,12]$ for rotations about the layer normal (Sec. II). From this, we derive the phase diagrams and structural organization when the or-

1799 (c) 1999 The American Physical Society 
der parameter is nonzero. The possibility of finding a large number of distinct phases (like the Devil's staircase and consistent with experimental observations of periods three times the layer spacing) will be demonstrated in Sec. V.

\section{A MODEL FREE ENERGY}

We begin by considering the the $\mathrm{Sm}-C^{*}$ phase, which we choose to view as a system of layer-averaged tilt vectors. The tilt vectors are constrained to point perpendicular to the layer normal and the magnitude of the tilt vector is equal to the sine of the tilt angle. To simplify the model, we immediately assume that this is a bulk system so that surfaces can safely be ignored. Further, we will work in the mean-field limit 'which assumes' that the layers are completely uniform so that all the gradient terms in the free energy are identically zero. We now will demonstrate how the ferriclinic phases can be modeled as a series of helical modulations of the average tilt vector. These phases are what we collectively refer to as heliclinic phases, since the tilt vector rotates in a helical fashion along the $z$ axis (the layer normal).

The Landau free energy of a system is constructed by summing together all the symmetry-allowed combinations of the average tilt vector per layer multiplied by some set of (phenomenological) Landau coefficients. Constructing the Landau free energy for our model system up to fourth order, we find

$$
\begin{aligned}
F= & \sum_{j \leqslant i} A_{i, j}^{(1)}\left(\vec{S}_{i} \cdot \vec{S}_{j}\right)+A_{i, j}^{(2)}\left(\vec{S}_{i} \times \vec{S}_{j}\right) \cdot \hat{\vec{z}} \\
& +\frac{1}{2} \sum_{j \leqslant i} \sum_{l \leqslant k} \dot{B}_{i, j, k, l}\left(\vec{S}_{i} \cdot \vec{S}_{j}\right)\left(\vec{S}_{k} \cdot \vec{S}_{l}\right)+O\left(S^{6}\right),
\end{aligned}
$$

where the sums range over all layers in the system. The vector $\vec{S}_{l}$ is the average tilt vector for the $l$ th layer. While this is a break with convention (smectic tilt vectors are normally written as $\vec{c}$ ), we will use $\vec{S}$ throughout this paper to help draw out the similarities between this model and simple magnetic models. The $A_{i, j}^{(1)}$ and the $A_{i, j}^{(2)}$ are, by convention, assumed to vary with temperature while the $B_{i, j, k, l}$ are considered constants. Further, we assume that the $A$ 's and the $B$ 's are short ranged, which is what is observed experimentally. The chiral term $\left(\vec{S}_{i} \cdot \vec{S}_{j}\right)\left(\vec{S}_{k} \times \vec{S}_{l}\right)$ is also symmetry allowed, but we assume that this term is small and may be

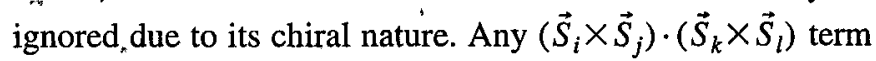
is nonchiral and can be transformed into terms we have al $\vec{s}$ ready included. If we limit interactions to only next-nearest neighbors, then the second order terms in Eq. (1) are the same as those in the free energy discussed by Čepič and Žekš [11]. In this paper, however, we allow for fourth order terms beyond the $(\vec{S} \cdot \vec{S})^{2}$ term used in [11]. These terms provide an important coupling between specific Fourier modes and must be included in a proper analysis.

Taking the Fourier transform of Eq. (1),

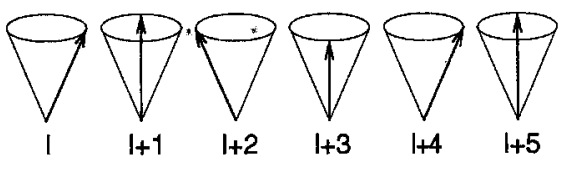

FIG. 1. Example of a heliclinic phase $(k=2 \pi / 4 a$ in this example).

$$
\begin{aligned}
F= & \sum_{k} a_{k}\left(\vec{S}_{k} \cdot \vec{S}_{-k}\right)+b_{k}\left(\vec{S}_{k} \cdot \vec{S}_{-k}\right)^{2} \\
& +\sum_{k} \sum_{k^{\prime}} \sum_{k^{\prime \prime} \neq k} c_{k, k^{\prime}, k^{\prime \prime}}\left(\vec{S}_{k} \cdot \vec{S}_{k^{\prime}}\right)\left(\vec{S}_{k^{\prime \prime}} \cdot \vec{S}_{-k-k^{\prime}-k^{\prime \prime}}\right) \\
& +O\left(S^{6}\right),
\end{aligned}
$$

where the sum is over wave vectors $k=2 \pi / n a, n= \pm 1$, $\pm 2, \pm 3, \ldots$, and $a$ is the smectic layer spacing (see Fig. 1). The sixth order terms are expected to be small and will not be considered further. Defining ' $q=2 \pi / a$, it is easily seen that $\vec{S}_{i q}$ is the ferroclinic order parameter of the smectic $(\vec{S}$ is an $X Y$ spin), while $\vec{S}_{q / 2}$ is the antiferroclinic order parameter. What we call $\vec{S}_{q}$ is often written $\vec{S}_{0,}$ but to better illustrate the relationship between the different wave vectors, we will always refer to the ferroclinic order parameter as $\vec{S}_{q}$. The next term, $\vec{S}_{q / 3}$, we call the heliclinic order parameter, which is similar to the order parameter for the planar helimagnetic phases observed in some rare-earth magnetic materials such as Terbium [13]. The fourth order term $\left(\vec{S}_{q} \cdot \vec{S}_{-q / 3}\right)\left(\vec{S}_{-q / 3} \cdot \vec{S}_{-q / 3}\right)$ provides a direct coupling (mode locking) between the $\vec{S}_{q}$ and $\vec{S}_{q / 3}$ order parameters. We will show below that the phase of this term can always be chosen so that this term has a negative coefficient and thus if $\vec{S}_{q / 3} \cdot$ is present it will become energetically favorable for $\vec{S}_{q}$ to have a nonzero magnitude as well. This mode locking extends the range over which it is energetically favorable to have a ferroclinic component. This extra stabilizing term has important consequences when we construct the phase diagram. Because additional terms are likely to be small and because we wish to avoid the difficulties inherent in a full-blown field theory, we will assume from here on that we are working in a region of the phase diagram where we do not need to consider Fourier components with $n>3$. At this level of approximation, there are no other mode lockings between different Fourier modes.

Explicitly writing out the summation up to $n=3$, we' find

$$
F=F_{0}+F_{\text {int }},
$$

where

$$
\begin{aligned}
F_{0}= & a_{1}\left(\vec{S}_{q} \cdot \vec{S}_{-q}\right)+a_{2}\left(\vec{S}_{q / 2} \cdot \vec{S}_{-q / 2}\right)+a_{3}\left(\vec{S}_{q / 3} \cdot \vec{S}_{-q / 3}\right) \\
& +b_{1}^{\prime}\left(\vec{S}_{q} \cdot \vec{S}_{-q}\right)^{2}+b_{2}^{\prime}\left(\vec{S}_{q / 2} \cdot \vec{S}_{-q / 2}\right)^{2}+b_{3}^{\prime}\left(\vec{S}_{q / 3} \cdot \vec{S}_{-q / 3}\right)^{2} \\
& +c_{12}^{\prime}\left(\vec{S}_{q} \cdot \vec{S}_{-q}\right)\left(\vec{S}_{q / 2} \cdot \vec{S}_{-q / 2}\right)+c_{13}^{\prime}\left(\vec{S}_{q} \cdot \vec{S}_{-q}\right)\left(\vec{S}_{q / 3} \cdot \vec{S}_{-q / 3}^{\prime}\right) \\
& +c_{23}^{\prime}\left(\vec{S}_{q / 2} \cdot \vec{S}_{-q / 2}\right)\left(\vec{S}_{q / 3} \cdot \vec{S}_{-q / 3}\right)
\end{aligned}
$$




$$
\begin{aligned}
F_{\text {int }}= & b_{1}^{\prime \prime}\left(\vec{S}_{q} \cdot \vec{S}_{q}\right)\left(\vec{S}_{-q} \cdot \vec{S}_{-q}\right)+b_{2}^{\prime \prime}\left(\vec{S}_{q / 2} \cdot \vec{S}_{q / 2}\right)\left(\vec{S}_{-q / 2} \cdot \vec{S}_{-q / 2}\right) \\
& +b_{3}^{\prime \prime}\left(\vec{S}_{q / 3} \cdot \vec{S}_{q / 3}\right)\left(\vec{S}_{-q / 3} \cdot \vec{S}_{-q / 3}\right)+c_{12}^{\prime \prime}\left(\vec{S}_{q} \cdot \vec{S}_{-q / 2}\right) \\
& \times\left(\vec{S}_{-q} \cdot \vec{S}_{q / 2}\right)+c_{13}^{\prime \prime}\left(\vec{S}_{q} \cdot \vec{S}_{-q / 3}\right)\left(\vec{S}_{-q} \cdot \vec{S}_{q / 3}\right) \\
& +c_{23}^{\prime \prime}\left(\vec{S}_{q / 2} \cdot \vec{S}_{-q / 3}\right)\left(\vec{S}_{-q / 2} \cdot \vec{S}_{q / 3}\right)+c_{12}^{\prime \prime \prime}\left(\vec{S}_{q} \cdot \vec{S}_{q / 2}\right) \\
& \times\left(\vec{S}_{-q} \cdot \vec{S}_{-q / 2}\right)+c_{13}^{\prime \prime \prime}\left(\vec{S}_{q} \cdot \vec{S}_{q / 3}\right)\left(\vec{S}_{-q} \cdot \vec{S}_{-q / 3}\right) \\
& +c_{23}^{\prime \prime \prime}\left(\vec{S}_{q / 2} \cdot \vec{S}_{q / 3}\right)\left(\vec{S}_{-q / 2} \cdot \vec{S}_{-q / 3}\right)+\frac{c_{1333}}{2}\left[\left(\vec{S}_{q} \cdot \vec{S}_{-q / 3}\right)\right. \\
& \left.\times\left(\vec{S}_{-q / 3} \cdot \vec{S}_{-q / 3}\right)+\left(\vec{S}_{-q} \cdot \vec{S}_{q / 3}\right)\left(\vec{S}_{q / 3} \cdot \vec{S}_{q / 3}\right)\right] .
\end{aligned}
$$

Since $\vec{S}_{k}$ is the Fourier transform of a real function, $\vec{S}_{k}{ }^{*}$ $=\vec{S}_{-k}$ and so we can immediately rewrite Eq. (4) as

$$
\begin{aligned}
F_{\text {int }}= & b_{1}^{\prime \prime}\left|\vec{S}_{q} \cdot \vec{S}_{q}\right|^{2}+b_{2}^{\prime \prime}\left|\vec{S}_{q / 2} \cdot \vec{S}_{q / 2}\right|^{2}+b_{3}^{\prime \prime}\left|\vec{S}_{q / 3} \cdot \vec{S}_{q / 3}\right|^{2} \\
& +c_{12}^{\prime \prime}\left|\vec{S}_{q} \cdot \vec{S}_{-q / 2}\right|^{2}+c_{13}^{\prime \prime}\left|\vec{S}_{q} \cdot \vec{S}_{-q / 3}\right|^{2}+c_{23}^{\prime \prime}\left|\vec{S}_{q / 2} \cdot \vec{S}_{-q / 3}\right|^{2} \\
& +c_{12}^{\prime \prime \prime}\left|\vec{S}_{q} \cdot \vec{S}_{q / 2}\right|^{2}+c_{13}^{\prime \prime \prime}\left|\vec{S}_{q} \cdot \vec{S}_{q / 3}\right|^{2}+c_{23}^{\prime \prime \prime}\left|\vec{S}_{q / 2} \cdot \vec{S}_{q / 3}\right|^{2} \\
& +c_{1333} \operatorname{Re}\left\{\left(\vec{S}_{-q} \cdot \vec{S}_{q / 3}\right)\left(\vec{S}_{q / 3} \cdot \vec{S}_{q / 3}\right)\right\}
\end{aligned}
$$

\section{ORDER PARAMETERS}

The ferroclinic order parameter, as a real yalued $X Y$ spin, has two independent variables. These can be taken as the magnitude and direction of the vector. We define the $x$ axis of our coordinate system using the ferroclinic order parameter $\vec{S}_{q}$

$$
\vec{S}_{q}=\left|\vec{S}_{q}\right| \vec{x} \equiv X \vec{x}
$$

The antiferroclinic order parameter is also a real $X Y$ spin and so it too must have two independent variables. Let the first be the vector magnitude and the second be the coordinate system relative to the ferroclinic order parameter,

$$
\vec{S}_{q / 2}=\left|\vec{S}_{q / 2}\right|[\vec{x} \cos (\phi)+\vec{y} \sin (\phi)] \equiv Y[\vec{x} \cos (\phi)+\vec{y} \sin (\phi)]
$$

With the heliclinic order parameter, unlike with the ferroclinic or antiferroclinic order parameters, $+q / 3$ is not the same as $-q / 3$. Thus, the heliclinic order parameter is a complex $X Y$ spin with four free parameters. Parametrize this order parameter as

$$
\begin{aligned}
\vec{S}_{q / 3} & =\left|\vec{S}_{q / 3}\right|\left[\hat{x} \cos (\alpha) e^{i \psi}-i \vec{y} \sin (\alpha) e^{i \sigma}\right] \\
& =Z e^{i \psi}\left[\hat{\vec{x}} \cos (\alpha)-i \vec{y} \sin (\alpha) e^{i\left(\sigma^{+} \psi\right)}\right]
\end{aligned}
$$

where $Z, \alpha, \psi$, and $\sigma$ are the four independent variables.

Using the definitions (6)-(8), and substituting into Eqs. (3) and (5) we find

$$
\begin{aligned}
F_{0}= & a_{1} X^{2}+a_{2} \dot{Y}^{2}+a_{3} Z^{2}+b_{1}^{\prime} X^{4}+b_{2}^{\prime} Y^{4}+b_{3}^{\prime} Z^{4}+c_{12}^{\prime} X^{2} Y^{2} \\
& +c_{13}^{\prime} X^{2} Z^{2}+c_{23}^{\prime} Y^{2} Z^{2}
\end{aligned}
$$

and

$$
\begin{aligned}
F_{\text {int }}= & b_{1}^{\prime \prime} X^{4}+b_{2}^{\prime \prime} Y^{4} \cos ^{2}(2 \phi)+b_{3}^{\prime \prime} Z^{4}\left[1-\sin ^{2}(2 \alpha) \sin ^{2}\right. \\
& \times(\psi-\sigma)]+\left(c_{12}^{\prime \prime}+c_{12}^{\prime \prime \prime}\right) \cos ^{2}(\phi) X^{2} Y^{2}+\left(c_{13}^{\prime \prime}+c_{13}^{\prime \prime \prime}\right) \\
& \times \cos ^{2}(\alpha) X^{2} Z^{2}+\left(c_{23}^{\prime \prime}+c_{23}^{\prime \prime \prime}\right) Y^{2} Z^{2}\left\{\cos ^{2}(\phi-\alpha)\right. \\
& \left.-\sin (2 \phi) \sin (2 \alpha) \cos ^{2}[(\psi-\sigma) / 2]\right\}+c_{1333} X Z^{3} \cos (\alpha) \\
& \times\left[\cos ^{2}(\alpha) \cos (3 \psi)-\sin ^{2}(\alpha) \cos (2 \sigma+\psi)\right]
\end{aligned}
$$

The above free energy contains a large number of free parameters that must be found by minimization. This yields a large system of simultaneous equations that must be solved to produce a complete solution. In this paper we only consider the special case where antiferroclinic behavior is not present and consequently $Y=0$. Since there is no mode locking between the antiferroclinic component and any other $\vec{S}_{k}$ for $k \geqslant 2 \pi / 3$, this is the same as constraining $a_{2}, c_{12}^{\prime}$, and $c_{23}^{\prime}$ to be positive. These added constraints simplify the free energy considerably and we are left with

$$
F_{0}=a_{1} X^{2}+a_{3} Z^{2}+b_{1} X^{4}+b_{3} Z^{4}+c_{13}^{\prime} X^{2} Z^{2}
$$

and

$$
\begin{aligned}
F_{\text {int }}= & -b_{3}^{\prime \prime} Z^{4} \sin ^{2}(2 \alpha) \cos ^{2}(\psi-\sigma)+c_{13} \cos ^{2}(\alpha) X^{2} Z^{2} \\
& +c_{1333} X Z^{3} \cos (\alpha)\left[\cos ^{2}(\alpha) \cos (3 \psi)\right. \\
& \left.-\sin _{i}^{2}(\alpha) \cos (2 \sigma+\psi)\right]
\end{aligned}
$$

where we have defined

$$
\begin{gathered}
c_{13}=c_{13}^{\prime \prime}+c_{13}^{\prime \prime \prime}, \\
b_{1}=b_{1}^{\prime}+b_{1}^{\prime \prime}, \\
b_{3}=b_{3}^{\prime}+b_{3}^{\prime \prime} .
\end{gathered}
$$

Note that while the free energy does not explicitly depend on the sign of $\alpha$, the parameter $a_{3}$ must have an implicit dependence on $\alpha$ since a chiral system distinguishes between righthanded and left-handed modulations. We will return to this point later on when we construct the real-space orientations of the molecules.

Next we reparametrize $\sigma$ and $\psi$ by defining

$$
\begin{gathered}
\gamma=\sigma-\psi, \\
3 \delta=\sigma+2 \psi
\end{gathered}
$$

so that Eq. (12) becomes,

$$
\begin{aligned}
F_{\text {int }}= & -b_{3}^{\prime \prime} Z^{4} \sin ^{2}(2 \alpha) \cos ^{2}(\gamma)+c_{13} \cos ^{2}(\alpha) X^{2} Z^{2} \\
& +c_{1333} \dot{X} Z^{3} \cos (\alpha)[\cos (3 \delta) \cos (\gamma) \cos (2 \alpha) \\
& +\sin (3 \delta) \sin (\gamma)] .
\end{aligned}
$$

Since $\delta$ only appears in the final term of Eq. (15) we can quickly find the value of $\delta$ that minimizes the free energy 


$$
\frac{\partial F}{\partial(3 \delta)}=-\sin (3 \delta) \cos (\gamma) \cos (2 \alpha)+\cos (3 \delta) \sin (\gamma)=0
$$

\section{MODE LOCKING}

All the terms in Eq. (15) except for the $c_{1333}$ term are positive semidefinite functions of $\alpha$. Thus, the sign of $\cos (\alpha)$ is completely determined by this one term and consequently

$$
\begin{aligned}
& \cos (\alpha) X Z^{3} c_{1333}[\cos (3 \delta) \cos (\gamma) \cos (2 \alpha)+\sin (3 \delta) \sin (\gamma)] \\
& \quad \leqslant 0
\end{aligned}
$$

if $F$ is to have its minimal value. Let $\cos (\alpha)$ be such that

$$
\begin{aligned}
& -\cos (\alpha) c_{1333} X Z^{3} \mid \cos (3 \delta) \cos (\gamma) \cos (2 \alpha) \\
& +\sin (3 \delta) \sin (\gamma) \mid>0 .
\end{aligned}
$$

$X$ and $Z$ as the magnitude of vectors are intrinsically nonnegative. If we rewrite the absolute value as

$$
\begin{aligned}
& \left|\cos (3 \delta) \cos (\gamma) \cos (2 \alpha)_{x}+\sin (3 \delta) \sin (\gamma)\right| \\
& \quad=\sqrt{[\cos (3 \delta) \cos (\gamma) \cos (2 \alpha)+\sin (3 \delta) \sin (\gamma)]^{2}},
\end{aligned}
$$

then after expanding and substituting Eq. (16) into Eq. (19) to eliminate $\delta$, we find

$$
\begin{aligned}
& |\cos (3 \delta) \cos (\gamma) \cos (2 \alpha)+\sin (3 \delta) \sin (\gamma)| \\
& \quad=\sqrt{1-\sin ^{2}(2 \alpha) \cos ^{2}(\gamma)} .
\end{aligned}
$$

This yields the free energy

$$
\begin{gathered}
F=F_{0}+F_{\text {int }}, \\
F_{0}=a_{1} X^{2}+a_{3} Z^{2}+b_{1} X^{4}+b_{3} Z^{4}+c_{13}^{\prime} X^{2} Z^{2}, \\
F_{\text {int }}=-b_{3}^{\prime \prime} Z^{4} \sin ^{2}(2 \alpha) \cos ^{2}(\gamma)+c_{13} \cos ^{2}(\alpha) X^{2} Z^{2} \\
-c_{1333} X Z^{3} \cos (\alpha) \sqrt{1-\sin ^{2}(2 \alpha) \cos ^{2}(\gamma)} .
\end{gathered}
$$

The noninteracting portion of the free energy, $F_{0}$, behaves much like a magnet in the mean-field approximation and for arbitrary wave vector $k$ has the form

$$
\begin{aligned}
f_{k}= & a_{k}\left(\vec{S}_{k} \cdot \vec{S}_{-k}\right)+b_{k}\left(\vec{S}_{k} \cdot \vec{S}_{-k}\right)^{2}+\sum_{k^{\prime}} c_{k, k^{\prime}}\left(\vec{S}_{k} \cdot \vec{S}_{-k}\right) \\
& \times\left(\vec{S}_{k^{\prime}} \cdot \vec{S}_{-k^{\prime}}\right) .
\end{aligned}
$$

For any given mode $k$, the summed $c_{k, k^{\prime}}$ terms act as a perturbation to the quadratic $a_{k}$ term. This shifts the transition point in a nontrivial manner, but does not modify the underlying physics. The interaction term $F_{\text {int }}$, on the other hand, is responsible for (among other things) the mode locking between the $\vec{S}_{q}$ and $\vec{S}_{q / 3}$ modes, which stabilizes specific Fourier components. These restoring forces add phases to the phase diagram that do not have any analog in the simple magnetic systems modeled by $F_{0}$.

\section{PHASE DIAGRAM AND CHARACTERIZATION}

Since all the temperature dependence of the free energy (21) is assumed to be contained in $a_{1}$ and $a_{3}$, these two parameters determine the phase of the system. First, consider the parameter $\gamma$. Looking at the free energy (21), it is immediately apparent that if $b_{3}^{\prime \prime}<0$ then $\cos ^{2}(\gamma)=0$ always minimizes the free energy. If, on the other hand, $b_{3}^{\prime \prime}$ is positive, it is useful to consider a reparametrized free energy. Let

$$
\begin{gathered}
\zeta=\cos (\alpha), \\
\xi^{2}=\cos ^{2}(\gamma) \sin ^{2}(2 \alpha),
\end{gathered}
$$

and the free energy (21) becomes

$$
F=F_{0}-b_{3}^{\prime \prime} Z^{4} \xi^{2}+c_{13} X^{2} Z^{2} \zeta^{2}-c_{1333} X Z^{3} \zeta \sqrt{1-\xi^{2}} .
$$

First minimizing with respect to $\zeta$, we find

$$
\zeta=\frac{c_{1333} Z}{2 c_{13} X} \sqrt{1-\xi^{2}}
$$

From the definition of $\zeta$, if this results in $|\zeta|>1$ then the minimum must be $|\zeta|=1$. Assume for the time being that the equilibrium value of $\zeta$ is such that $|\zeta|<1$ since if $\zeta=1$ then $\xi^{2}=0$ from our definition of $\xi^{2}$ which implies that $\gamma$ is undetermined. Substituting back into the free energy,

$$
F=F_{0}-\frac{c_{1333^{2}}}{4 c_{13}} Z^{4}+\left(\frac{c_{1333}^{2}}{4 c_{13}}-b_{3}^{\prime \prime}\right) Z^{4} \xi^{2}
$$

By inspection, the equilibrium value of $\xi$ is

$$
\begin{aligned}
& \xi^{2}=0, \quad c_{1333}{ }^{2} / 4 c_{13}-b_{3}^{\prime \prime}>0, \\
& \xi^{2}=\infty, \quad c_{1333}{ }^{2} / 4 c_{13}-b_{3}^{\prime \prime}<0 .
\end{aligned}
$$

Since mathematically $|\zeta| \leqslant 1$, however, $\xi^{2}$ can never exceed unity. Looking back on the definitions of $\zeta$ and $\xi$, the proper interpretation of this result is that $\cos ^{2}(\gamma)$ takes on only two values: zero or unity. The equilibrium value of $\cos ^{2}(\gamma)$ is the one that results in the lower free energy (24).

First, consider the case when $\cos ^{2}(\gamma)=0$, whose phase diagram is summarized in Fig. 2. Minimizing the free energy (21) yields a system of equations:

$$
\begin{aligned}
\frac{\partial F}{\partial X}= & 0 \\
= & a_{1} X+2 b_{1} X^{3}+X Z^{2}\left[c_{13}^{\prime}+c_{13} \cos ^{2}(\alpha)\right] \\
& -\frac{1}{2} c_{1333} \cos (\alpha) Z^{3},
\end{aligned}
$$




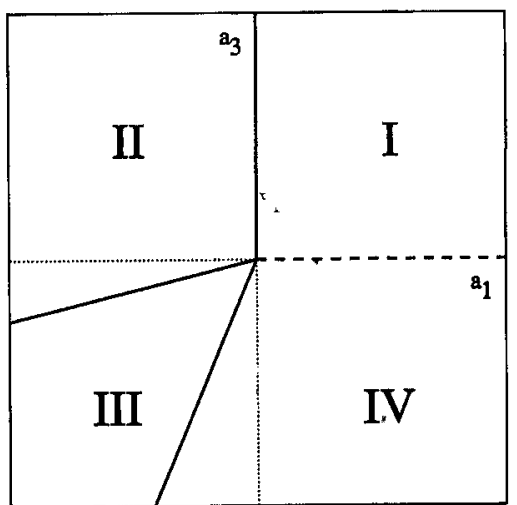

FIG. 2. Phase diagram for $b_{3}^{\prime \prime}<0$. Phase I' is the isotropic phase, II is the ferroclinic, III is the ferriclinic $0<\cos (\alpha)<1$ phase, and IV is the ferriclinic $\cos (\alpha)=1$ phase. Dotted lines are coordinate axis, solid lines are continuous transitions, heavy dashed lines are discontinuous transitions.

$$
\begin{aligned}
\frac{\partial F}{\partial Z}= & 0 \\
= & Z\left(a_{3}+2 b_{3} Z^{2}+X^{2}\left[c_{13}^{\prime}+c_{13} \cos ^{2}(\alpha)\right]\right. \\
& \left.-\frac{3}{2} c_{1333} X Z \cos (\alpha)\right) \\
\frac{\partial F}{\partial \alpha} & =0=X Z^{2}\left[2 c_{13} X \cos (\alpha)-c_{1333} Z\right]
\end{aligned}
$$

Since $X$ and $Z$ are the magnitudes of vectors and hence must be non-negative, for $a_{1}>0$ and $a_{3}>0$ the system is in an isotropic phase with

$$
X=Z=\cos (\alpha)=0
$$

Since it has no tilt, this phase must be the Sm-A phase of the liquid crystals. Exactly at the point $a_{1}=a_{3}=0$, the quadratic terms in the free energy vanish and there is a critical point.

Next, consider the quadrant: where $a_{1}<0$. Since $a_{1}$ is negative, we expect that $X$ will be nonzero. For large enough values of $a_{3}$, one always finds $Z=0$ and as a consequence $\cos (\alpha)$ is unspecified. Equations (25) and (26), however, are easily solved. Anticipating the case when $Z \neq 0$, we assign $\cos (\alpha)=0$ and so

$$
\begin{gathered}
X^{2}=-a_{1} / 2 b_{1}, \\
Z^{2}=0, \\
\cos (\alpha)=0,
\end{gathered}
$$

which are the results one would expect for a simple ferromagnet or, in our case, the ferroclinic phase.

As $a_{3}$ decreases, $Z$ eventually becomes nonzero. Whether this happens for $a_{3}$ positive or negative is determined by the sign of $c_{13}^{\prime}$. Equations (25)-(27) are once again easily solved and we now find

$$
\begin{gathered}
X^{2}=\frac{a_{3} c_{13}^{\prime}-2 a_{1}\left[b_{3}-\left(c_{1333}^{2} / 4 c_{13}\right)\right]}{4 b_{1}\left[b_{3}-\left(c_{1333}{ }^{2} / 4 c_{13}\right)\right]-c_{13}^{\prime 2}} \\
Z^{2}=\frac{a_{1} c_{13}^{\prime}-2 a_{3} b_{1}}{4 b_{1}\left[b_{3}-\left(c_{1333}^{2} / 4 c_{13}\right)\right]-c_{13}^{\prime 2}} \\
\cos (\alpha)=c_{1333} Z / 2 c_{13} X .
\end{gathered}
$$

Note that $\cos (\alpha)$ is proportional to $Z$, which justifies my previous statement that $\cos (\alpha)=0$ when $Z=0$. From Eq. (32), the phase transition from $Z^{2}=0$ to $Z^{2}>0$ must occur when

$$
a_{3}=\frac{c_{13}^{\prime}}{2 b_{1}} a_{1}
$$

When $\cos (\alpha)=1$, the system undergoes a continuous transition to a new phase, since the value of $\cos (\alpha)$ is unable to increase further. Setting Eq. (32) equal to unity, this transition occurs when

$$
a_{1}=\frac{2 b_{1} c_{1333}{ }^{4}+4 c_{13}^{\prime} c_{13}^{2}}{8 b_{3} c_{13}^{2}-2 c_{13} c_{1333}{ }^{2}+c_{13}^{\prime} c_{1333}{ }^{4}} a_{3} .
$$

In this phase, the equilibrium values of $X$ and $Z$ are given by

$$
\begin{gathered}
0=a_{1} X+2 b_{1} X^{3}+\left(c_{13}^{\prime}+c_{13}\right) X Z^{2}-c_{1333} Z^{3} / 2, \\
0=a_{3} Z+2 b_{3} Z^{3}+\left(c_{13}^{\prime}+c_{13}\right) X^{2} Z-3 c_{1333} X Z^{2} / 2 .
\end{gathered}
$$

While of simple form, these equations are difficult to solve for arbitrary coefficients and we will leave them as they are.

While it might appear that a stable phase with $X=0, Z$ $\neq 0$ is possible, this is not-the case. When $X=0$, minimizing the free energy in Eq. (25) requires that $\cos (\alpha)=0$. Physically, however, when $X=0$, the coordinate system is no longer defined and so, by extension, $\vec{S}_{q / 3}$ must be rotationally invariant. From the definition of $\vec{S}_{q / 3}$ in Eq. (8) and setting $\cos (\alpha)=0$, one finds that

$$
\vec{S}_{q / 3}=-i Z \vec{y}
$$

which is not rotationally invariant. Further, looking at the stability of the free energy at that point we see that

$$
\left.\frac{\partial^{2} F}{\partial \cos (\alpha)^{2}}\right|_{\cos (\alpha)=0}=2 c_{13} X^{2} Z^{2}
$$

which is zero when $X=0$. This implies an inflection point in the free energy which is incompatible with the requirement from Eq. (25) that $\cos (\alpha)=0$ be a minimum when $X=0$. Hence, we conclude that no additional phases can appear so long as $\cos ^{2}(\gamma)$ remains zero. Thus as a consequence of the mode locking between $\vec{S}_{q / 3}$ and $\vec{S}_{q}$ we find that a ferroclinic component is present even when all terms containing $X^{2}$ in the free energy are positive.

Minimizing the free energy when $\cos ^{2}(\gamma)=1$, on the other hand, yields a system of coupled equations 


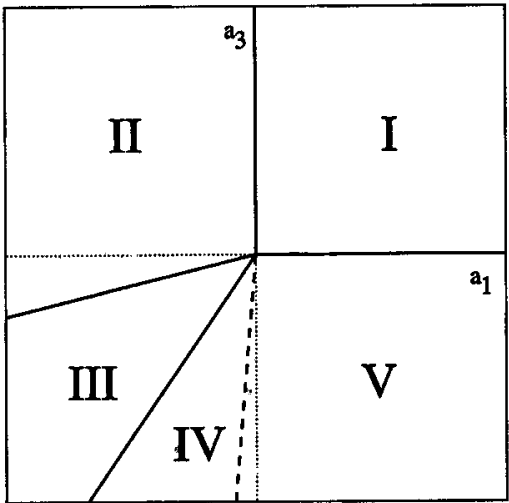

FIG. 3. Phase diagram for $b_{3}^{\prime \prime}>0$. Phase I is the isotropic phase, II is the ferroclinic, III is the ferriclinic $0<\cos (\alpha)<1$ phase, IV is the ferriclinic $\cos (\alpha)=1$ phase, and $\mathrm{V}$ is the pure helix $(X=0)$ phase. Depending on the sign of $\Delta F$, region IV may or may not be present in à given system. Dotted lines are coordinate axis, solid lines are continuous transitions, heavy dashed lines are discontinuous transitions.

$$
\begin{aligned}
\frac{\partial F}{\partial X}= & 0 \\
= & a_{1} X+2 b_{1} X^{3}+X Z^{2}\left[c_{13}^{\prime}+c_{13} \cos ^{2}(\alpha)\right] \\
& -\frac{1}{2} c_{1333} \cos (\alpha) Z^{3}|\cos (2 \alpha)|
\end{aligned}
$$

$$
\begin{aligned}
\frac{\partial F}{\partial Z}= & 0 \\
= & a_{3} Z+2 b_{3} Z^{3}+X^{2} Z\left[c_{13}^{\prime}+c_{13} \cos ^{2}(\alpha)\right] \\
& -\frac{3}{2} c_{1333} \cos (\alpha) X Z^{2}|\cos (2 \alpha)|-2 b_{3}^{\prime \prime} Z^{3} \sin ^{2}(2 \alpha),
\end{aligned}
$$

$$
\begin{aligned}
\frac{\partial F}{\partial \alpha}= & 0 \\
= & 8 b_{3}^{\prime \prime} Z^{4} \cos (\alpha) \cos (2 \alpha)+2 c_{13} X^{2} Z^{2} \cos (\alpha) \\
& -c_{1333} X Z^{3}\left(|\cos (2 \alpha)|+4 \frac{\cos ^{2}(\alpha) \cos (2 \alpha)}{|\cos (2 \alpha)|}\right) .
\end{aligned}
$$

These equations correspond to the phase diagram summarized in Fig. 3. If $Z=0$, then these equations reduce to the $\cos ^{2}(\gamma)=0$ case and we recover the solution for a Sm-A phase in Eq. (28) and for a ferroclinic phase in Eq. (29).

It is known from basic thermodynamics that the system undergoes a phase transition when the free energy with $\cos ^{2}(\gamma)=1$ is equal to the free energy with $\cos ^{2}(\gamma)=0$ because the functional form of the free energy changes abruptly at that point. Let

$$
\left.\Delta F \equiv F\right|_{\cos ^{2}(\gamma)=1}-\left.F\right|_{\cos ^{2}(\gamma)=0} .
$$

The transition from $\cos ^{2}(\gamma)=0$ to $\cos ^{2}(\gamma)=1$ occurs when $\Delta F \leqslant 0$.

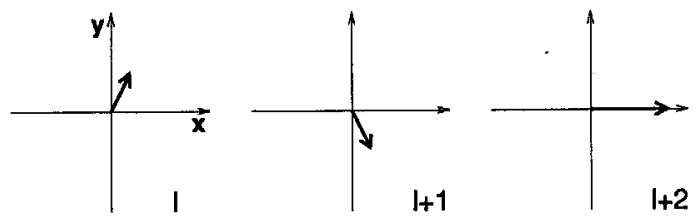

FIG. 4. Phase III, $\cos ^{2}(\gamma)=0[X \neq 0, Z \neq 0,0<\cos (\alpha)<1]$. The resulting tilt vector, $\vec{c}_{\ell}$, is plotted for $\ell=1,2,3$.

When $Z \neq 0$, the system is in either the $\cos ^{2}(\gamma)=0$ phase we looked at above Eq. (32) or it is in a new $\cos ^{2}(\gamma)=1$ phase, depending on the sign of $\Delta F$ in Eq. (42). In the $\cos ^{2}(\gamma)=1$ phase, the full set of Eqs. (39)-(41) must be solved to completely determine the phase diagram of the system. A few solutions, however, can be obtained by inspection. First, if $\cos (\alpha)=1$, then the system (39), (40) becomes identical to (25), (26) and we conclude that in this region $\cos ^{2}(\gamma)$ is undetermined, which we could have inferred from the definition of $\vec{S}_{q / 3}$ in Eqs. (8) and (14).

On the other hand, when $X=0$ it is immediately apparent that

$$
\begin{gathered}
X=0 \\
Z=-a_{3} / 2\left(b_{3}-b_{3}^{\prime \prime}\right) \\
\cos (\alpha)=1 / \sqrt{2}
\end{gathered}
$$

is the solution.

Finally, if $\cos (\alpha)=0$ and $a_{1}>0$ then the free energy once again takes on the functional form of a magnet in a mean field and we are left with

$$
\begin{gathered}
X=0, \\
Z=-a_{3} / 2 b_{3}, \\
\cos (\alpha)=0 .
\end{gathered}
$$

However, since we know that $b_{3}^{\prime \prime}$ is positive [or else $\cos ^{2}(\gamma)$ would be identically zero], the solution given in Eq. (43) always has a lower free energy than Eq. (44). Consequently, Eq. (44) is unstable with respect to Eq. (43).

Numerical investigations (assigning order of magnitude estimates to the various constant parameters) indicate that for some range of parameters, there is also an intermediate $X$ $\neq 0, Z \neq 0,0<\cos (\alpha)<\cos (\pi / 4)$ phase with $\cos ^{2}(\gamma)=1$. The analytic solution, however, is sufficiently difficult that we are unable to investigate this phase any further.

Now that we have completed an outline of the phase diagram both for $\cos ^{2}(\gamma)=0$ (Fig. 2) and for $\cos ^{2}(\gamma)=1$ (Fig. 3), let us examine what this says about the molecular orientation within the smectic layers. If $\cos ^{2}(\gamma)=0$, then from the definition (14) of $\gamma$ we find

$$
\sigma=\psi+n \pi
$$

and so from the definition of $\vec{S}_{q / 3}$ in Eq. (8)

$$
\begin{aligned}
\vec{S}_{q / 3} & =Z e^{i \psi}\left[\vec{x} \cos (\alpha)-i \vec{y} e^{i n \pi} \sin (\alpha)\right] \\
& =Z e^{i \psi}[\vec{x} \cos ( \pm \alpha)-i \vec{y} \sin ( \pm \alpha)],
\end{aligned}
$$



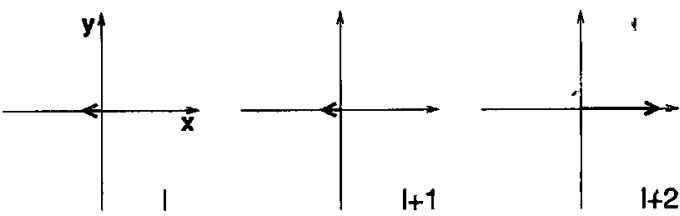

FIG. 5. Phase IV $[X \neq 0, Z \neq 0, \cos (\alpha)=1]$. The resulting tilt vector, $\vec{c}_{\bullet \ell}$, is plotted for $\ell=1,2,3$.

which, given the constraint on $\cos (\alpha)$ in Eq. (18), minimizes $F$ when

$$
\operatorname{Re}\left\{e^{i \psi}\right\}=+1
$$

Physically, this means that the heliclinic order parameter $\vec{S}_{q / 3}$ is completely in phase (modulg, $2 \pi / 3$ ) with the ferroclinic order parameter $\vec{S}_{q}$. The ambiguity in the, sign of $\alpha$ occurs because $\cos (\alpha)$ is an even function of $\alpha$ and we have not specified how $a_{3}$ depends on the sign of the chiral pitch. For convenience, we assume for the rest of this paper that $a_{3}$ varies with the chirality such that $+\alpha$ is the lower energy solution.

To find the real-space tilt 'vector, just sum the Fourier series

$$
\begin{aligned}
\vec{S}_{\ell}= & \sum_{k}\left(e^{-i \ell k / n} \vec{S}_{k}\right)=[X+Z \cos (\alpha) \cos (2 \pi \ell / 3)] \vec{x} \\
& +Z \sin (\alpha) \sin (2 \pi \ell / 3) \hat{y}_{*}
\end{aligned}
$$

When $|\cos (\alpha)|<1$, both $X$ and $Z$ are nonzero and the system is in the two order parameter phase as sketched in Fig. 4. When $\cos (\alpha)=1$, the $y$ component in Eq. (49) vanishes and both $\cos ^{2}(\gamma)=0$ and $\cos ^{2}(\gamma)=1$ take on the same functional form,

$$
\vec{S}_{\ell}=\left[X+Z_{1} \cos (2 \pi \ell / 3)\right] \vec{x}
$$

"This form, with its planar three-layer, unit cell and nonzero spontaneous polarization (see Fig. 5), is quite similar to that proposed by Takezoe et al. [4] for the $\mathrm{Sm}-C_{\gamma}$ phase.

In the $\cos ^{2}(\gamma)=1$ phase(s), on the other, hand, from the definition of $\gamma$ we find

$$
\sigma=\psi+n \pi / 2 .
$$

Substituting into Eq. (8) as we did tó get Eq. (45), we now find that

$$
\begin{gathered}
\vec{S}_{q / 3}=Z e^{i \psi}\left[\hat{x} \cos (\hat{\alpha})-i \hat{y} e^{n \pi / 2} \sin (\alpha)\right] \\
=Z e^{i \psi}[\hat{x} \cos ( \pm \alpha)+\hat{y} \sin ( \pm \alpha)]
\end{gathered}
$$

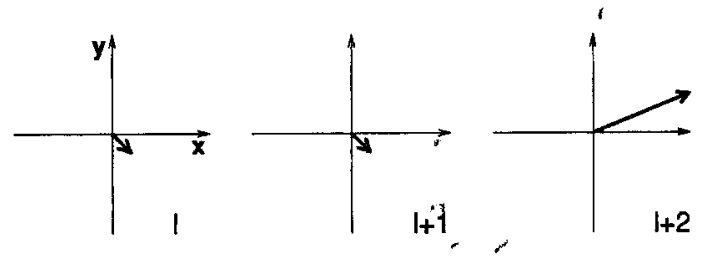

EIG. 6. Phase III, $\cos ^{2}(\gamma)=1[X \neq 0, Z \neq 0,0<\cos (\alpha)<1]$. The resulting tilt vector, $\vec{c}_{\ell}$, is plotted for $\ell=1,2,3$.

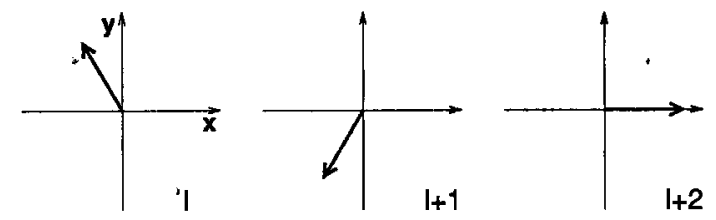

FIG. 7. Phase $\mathrm{V}\left[X=0, Z \neq 0, \sin ^{2}(2 \alpha)=1\right]$. The resulting tilt vector, $\vec{c}_{\ell}$, is plotted for $\ell=1,2,3$.

with $X, Z$, and $\alpha$ given by the system of equations 'in Eqs. (39)-(41). The net effect is to change the phase of the $y$ component of $\vec{S}_{q / 3}$ so that instead of $\vec{S}_{\ell} \cdot \vec{y} \propto \sin (2 \pi \ell / 3)$,

$$
\vec{S}_{\ell}=[X+Z \cos (\alpha) \cos (2 \pi \ell / 3)] \vec{x}+Z \sin (\alpha) \cos (2 \pi \ell / 3) \vec{y}
$$

$$
\therefore
$$

as shown in Fig. 6.

When $\sin (2 \alpha)=1$, the ferroclinic order parameter vanishes

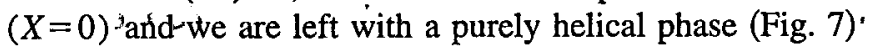

$$
\vec{S}_{\ell}^{r}=Z[\cos (2 \pi \ell / 3+\psi) \vec{x}+\sin (2 \pi \ell / 3+\psi) \hat{y}]
$$

where $\psi$ is an arbitrary phase. Since $\psi$ is now a gauge variable this implies the existence of a spontaneously broken symmetry with its corresponding Goldstone mode, just as the spontaneously broken symmetry of the $\mathrm{Sm}-C^{*}$ gives rise to a Goldstone mode in that phase.

\section{ANALYSIS}

In this paper, we focused on terms in the:free.energy up to $O\left(S^{4}\right)^{4}$ and only considered the Fourier modes $\vec{S}_{q}$ and $\vec{S}_{q / 3}$. We did this both to simplify the problem and because we only expect these two Fourier modes to have large' magnitudes. Consequently, they should be the easiest to' measure experimentally. Despite this simplified description, we find a two order parameter region, a mode locked phase, and two single order parameter phases, as shown in Table I. These phases are qualitatively similar to many of the phases observed experimentally. In particular, Fig. 5 bears a strong resemblance to the form of the $\mathrm{Sm}-C_{\gamma}$ proposed by Takezoe et al. [4]. This model is thus able to explain a much greater range of phase behaviors than the phenomenological model of Cepič and Žekš [11]. Our results, however, still do not provide a complete picture of the various $\mathrm{Sm}-C^{*}$ subphases due to the absence of the antiferroclinic order parameter. The antiferroclinic phases are known to play an important rolè

TABLE I. Summary of the different heliclinic phases and the order parameter(s) associated with each.

\begin{tabular}{lcccc}
\hline \hline Phase & Description & $X$ & $Z$ & "Polarization" \\
\hline I & Paraclinic $\left(\mathrm{Sm}-A^{*}\right)$ & 0 & 0 & N/A \\
\hline II & Ferroclinic $\left(\mathrm{Sm}-C^{*}\right)$ & $>0$ & 0 & linear \\
\hline III & Heliclinic-two order param. & $>0$ & $>0$ & elliptical \\
\hline IV & Heliclinic-mode locked & $>0$ & $>0$ & linear \\
\hline V & Heliclinic & 0 & $>0$ & circular \\
\hline
\end{tabular}


both in the phase diagram of materials such as MHPOBC. Solving the model in the presence of all three order parameters, however, is beyond the scope of this paper.

To construct an even more complete theory, especially one that includes modes whose wave vectors are not rational fractions of the zone boundary wave vector, several additional effects must be considered.First, it is natural to expect that these phases (like every other smectic phase with inplane rotation order) should, in a chiral system, have a slow twist of the axis associated swith their long-range orientational order. Mode locking occurs when the total wave vector (including the natural chirality) for specific terms in the free energy sum to zero.

Second, it is natural to expect that $\vec{S}_{q / 3}$, in general, has a somewhat lower susceptibility (i.e., a less negative value of $a_{k}$ ) than the neighboring values of the wave vector $k$. Thus, we expect that this mode locked phase will appear only if the energy decrease associated with the mode locking exceeds that associated with the smaller value of $a$. A detailed treatment of this is complicated and involves several additional parameters. Roughly speaking, however, the energy associated with a phase with $k$ close to $q / 3$ is $-a_{k}^{2} / 4 b_{3}$. That of the mode locked phase is approximately $-a_{3}^{2} / 4 b_{3}-f_{m}$ where $f_{m}$ is the value of the mode locking $c_{1333}$ term for the phases being considered. Thus mode locking is expected when

$$
\left(a_{m} / a_{3}\right)^{2} \leqslant 4 b_{3} f_{m}
$$

Here $a_{m}$ is the most negative value of $a_{k}$ for all $k$.

Next, it is also possible for there to be mode locked phases in which several modes, each with different values of $k_{\text {s }}$ happen to add up to $q=2 \pi n$. We believe that these are implausible (except for the slight helical complications discussed above), at least provided that the curvature of $a_{k}$ near $k=q / 3$ is positive.

Finally, Fourier modes with $k$ 's smaller than $2 \pi / 3$ will also experience mode locking once the higher order modes are included in the free energy. For example, $\left|\vec{S}_{q / 4} \cdot \vec{S}_{q / 4}\right|^{2}$ and $\left(\vec{S}_{q / 6} \cdot \vec{S}_{q / 6}\right)\left(\vec{S}_{q / 6} \cdot \vec{S}_{-q / 2}\right)$ are valid terms that also support mode locking (the first 'one is allowed as an Umklapp process). The gauge freedom we saw in the pure twist phase (51) represents the relative phase difference between that particular mode and some arbitrary reference.coordinate system (in our case, the $\vec{S}_{q}$ mode). Since this gauge freedom is present in every Fourier component but does not change the noninteracting free energy $F_{0}$, we can always assign to it a value such that mode locking lowers the energy of the system. Thus, the mode locking terms in the free energy then will combine to form an infinite sequence of lockins between modes $\vec{S}_{q / m}$ and $\vec{S}_{q / n}$ for every rational number $m / n$. Therefore we expect that, on expanding the free energy to all orders, mode locking will turn the continuous model for the ferriclinic phases of Cepič and Žekš [11] into a Devil's staircase, much like the model proposed by Takanishi et al. [7]. Our Devil's staircase, however, assumes the existence of short period helical modulations about the layer normal, which we believe better captures the physics of the ferriclinic phases.
We have recently become acquainted with a preprint by Mach et al. [6] of an experimental study they have performed on $10 \mathrm{OTBBB} 1 \mathrm{M} 7$, a liquid crystal compound with two distinct ferriclinic phases $\left(\mathrm{Sm}-C_{F 11}\right.$ and $\left.\mathrm{Sm}-C_{F I 2}\right)$ in addition to $\mathrm{Sm}-C_{\alpha}$, ferroclinic, and antiferroclinic phases. By examining the $x$-ray scattering off of free-standing films, they have measured the superlattice associated with these different phases. They observe a four-layer superlattice in the Sm- $C_{F I 2}$ phase, a three-layer superlattice in the Sm- $C_{F I 1}$ phase, and 'a spacing incommensurate with the underlying lattice in the $\mathrm{Sm}-C_{\alpha}$ phase. This measurement is consistent with this model once the higher order modes (especially q/4) are included, although the interpretation of the $\mathrm{Sm}-C_{\alpha}$ phase in the context of this model is uncertain. Their measurement may also distinguish between this model and the model of Čepič and Žekš $[1,1]$. Where their model predicts one 'continuous tränsition, ours predicts a series of discrete jumps. In experiments, the system is seen to make discrete jumps in the period of the helical pitch, which suggests our model may be more appropriate for these phases.

\section{CONCLUSIONS}

Thus, we have shown that by starting with a simple description of a chiral smectic system we can derive a meaningful'free ęnergy and model a wide variety of ferroclinic, antiferroclinic, and ferriclinic phases. While similar in approach to several existing models for these phases, we include in our free energy fourth order terms that do not appear in the models considered previously [11,12]. These symmétry-allowed terms encourage mode locking between different' Fourier modes. This mode locking, in turn, causes significant qualitative chang's in the resulting phase diagram:

Even under the radical assumption that only the ferroclinic and "twist" order parameters can be nonzero, our model predicts a number of interesting phases. We find a phase with no tilt which, by construction, we identify with the 'smectic- $A$ phase. We also find a phase with only ferroclinic"order which we naturally identify as the smectic- $C^{*}$ phase. In addition, we find a number of ferrielectric phases. One (see Fig. 5) bears a strong resemblance to the smectic$C_{\gamma}$ phase. Another, which consists of a helical modulation with a period of three times the layer spacing (see Fig. 7), may have been experimentally observed by Mach et al. [6]. The other phases (Figs. 4-6), while clearly ferroclinic phases, seem not to correspond to any phases yet observed. Much like the model of Lorman et al. [12], our model also predicts a single Goldstone mode should be present in all the ordered phases. Additional phases [including an antiferroclinic phase (smectic- $C^{A}$ ) and antiferroclinic two-phase regions] can be found by including additional order parameters, but only at the expense of increasing analytical difficulty.

\section{ACKNOWLEDGMENT}

This research was supported by Grant No. DMR-8920147 from the National Science Foundation's Science and Technology Center for Advanced Liquid Crystalline OpticalıMaterials (ALCOM). 
[1] P. G. de Gennes and J. Prost, ${ }_{,}$The Physics of Liquid Crystals, , 2nd ed. (Oxford University Press, New York, 1993).

[2] S. Merino et al., Phys. Rev. E 54, 5169 (1997).

[3] T. Isozaki, Y. Suzuki, and I. Kawamura, Jpn. J. Appl. Phys., Part 2 30, L1573 (1991).

[4] H. Takezoe, J. Lee, Y. Ouchi, and A. Fukuda, Mol. Cryst. Liq. Cryst. 202, 85 (1991).

[5] E. Gorecka, A. D. L. Chandani, and Y. Ouchi, Jpn. J. Appl. Phys., Part 1 23, 131 (1990).

[6] P. Mach, R. Pindak, A.-M. Levelut, P. Barois, H. T. Ngyen,
C. C. Huang, and L. Furenlid, "Phys. Rev. Lett. 81, 1015 (1998).

[7] Y. Takanishi et al., Jpn. J. Appl. Phys., Part 1 31, 2023 (1991).

[8] R. Bruinsma and J. Prost, J. Phys. II 4, 1209 (1994).

[9] M. Yamăshita and S. Miyazima, Ferroelectrics 148, 1 (1993).

[10] B. Rovšek, 'M. Čepič, and B. Žekš, Phys. Rev. E 54, 3113 (1997).

[11] M. Čepič and B. Žekš, Liq. Cryst. 20, 29 (1996).

[12] V. L. Lorman, A. A. Bulbitch, and P. Toledano, Phys. Rev. E 49, 1367 (1994).

[13] T. Garel and P. Pfeuty, J. Phys. C 9, L245 (1976). 